\title{
Une remarque sur la méthode des points extrémaux de F. Leja
}

par J. Górsir (Kraków)

Introduction. Dans les travaux [1], [2] et [3] la solution du problème de Dirichlet a été construite au moyen de la méthode des points extrémaux de $F$. Leja pour un domaine situé dans le plan ou dans l'espace à 3 dimensions. La solution du problème $\mathrm{y}$ est obtenue en effectuant deux passages à la limite (voir (2) et (4)). Il est très intéressant de savoir s'il est possible d'obtenir la solution sans avoir recours au second passage à la limite (4). Je vais donner les conditions suffisantes pour que cette question admette une réponse affirmative. D'abord je vais rappeler quelques résultats connus.

Soit $E$ un ensemble borné et fermé de diamètre transfini (capacité) positif situé dans le plan et $f(z)$ une fonction réelle continue, définie sur E. Posons

$$
\omega_{\lambda}(z, \zeta)=|z-\zeta| \exp \{-\lambda[f(z)+f(\zeta)]\},
$$

où $z, \zeta \in E$ et $\lambda$ est un paramètre. Un système de points $\left\{\eta_{0}^{(n \lambda)}, \eta_{1}^{(n \lambda)}\right.$, $\left.\ldots, \eta_{n}^{(n \lambda)}\right\}=\eta_{\lambda}^{(n)}$ sera dit $n$-ième système de points extrémaux par rapport à la fonction (1) lorsque

$$
\prod_{0 \leqslant j<k \leqslant n} \omega_{\lambda}\left(\eta_{j}^{(n \lambda)}, \eta_{k}^{(n \lambda)}\right) \geqslant \prod_{0 \leqslant j<k \leqslant n} \omega_{\lambda}\left(\zeta_{j}, \zeta_{k}\right)
$$

pour chaque système de $n+1$ points $\zeta_{j}, j=0,1, \ldots, n$ de l'ensemble E. Posons

$$
\Delta_{j}^{(n)}=\prod_{\substack{k=0 \\ k \neq j}}^{n} \omega_{\lambda}\left(\eta_{j}^{(n \lambda)}, \eta_{k}^{(n \lambda)}\right), \quad j=0,1, \ldots, n
$$

et soit $\Delta_{0}^{(n)} \leqslant \Delta_{j}^{(n)}$ pour $j=0,1,2, \ldots, n$. Désignons par $L_{n}^{(j)}\left(z ; \eta \lambda^{(n)}\right)$ les polynômes de Lagrange

$$
L_{n}^{(j)}\left(z ; \eta_{\lambda}^{(n)}\right)=\prod_{\substack{k=0 \\ k \neq j}}^{n} \frac{z-\eta_{k}^{(n \lambda)}}{\eta_{j}^{(n \lambda)}-\eta_{k}^{(n \lambda)}}
$$


et formons les fonctions

$$
\Phi_{n}^{(0)}\left(z ; \eta_{\lambda}^{(n)}, \lambda f(z)\right)=L_{n}^{(0)}\left(z, \eta_{\lambda}^{(n \lambda)}\right) \cdot \exp \left[n \lambda f\left(\eta_{0}^{(n \lambda)}\right)\right] .
$$

Désignons par $E_{\lambda}$ l'ensemble des points d'accumulation de la suite triangulaire $\left({ }^{1}\right)$

$$
\begin{aligned}
& \eta_{0}^{(1 \lambda)}, \eta_{1}^{(1 \lambda)} \\
& \eta_{0}^{(2 \lambda)}, \eta_{1}^{(2 \lambda)}, \eta_{2}^{(2 \lambda)} \\
& \ldots \ldots \ldots
\end{aligned}
$$

On sait [1] que la limite

$$
\lim _{n \rightarrow \infty} \sqrt[n]{\left|\Phi_{n}^{(0)}\left(z ; \eta_{\lambda}^{(n)}, \lambda f(z)\right)\right|}=\Phi(z ; \lambda f)
$$

existe pour chaque point $z \notin E_{\lambda}$ et que la fonction $\log \Phi(z ; \lambda f)$ possède les propriétés suivantes:

$1^{\circ}$ Elle est harmonique en dehors de l'ensemble $E_{\lambda}$ le point $z=\infty$ exclu, où $\log \Phi(z ; \lambda f)$ a un pôle logarithmique.

$2^{\circ}$ Lorsque $z \notin E_{\lambda}$ et $z$ tend vers un point quelconque $z_{0} \epsilon E_{\lambda}$ on a

$$
\lim _{z \rightarrow z_{0}} \log \Phi(z ; \lambda f)=\lambda f\left(z_{0}\right) \text {. }
$$

Pour chaque point $z \epsilon E-E_{\lambda}$ on a

$$
\log \Phi(z ; \lambda f) \leqslant \lambda f(z) .
$$

$3^{\circ}$ Soit $E$ la frontiere commune de deux domaines $D$ et $D_{\infty}$, où $D_{\infty}$ contient le point $z=\infty$. Alors pour chaque point $z \epsilon . D$ la limite

$$
\lim _{\lambda \rightarrow 0} \frac{1}{\lambda} \log \Phi(z ; \lambda f)=\psi(z)
$$

existe. La fonction $\psi(z)$ est la solution du problème de Dirichlet pour le domaine $D$ avec la valeur $f(z)$ sur $E$.

On sait (voir [4] et [5]) qu'il existe des fonctions $f(z)$ pour lesquelles le passage à la limite (4) est inutile, par exemple lorsque $f(z)=\sqrt[k]{\left|w_{r}(z)\right|}$, $w_{r}(z)$ étant un polynôme de degré $r$ ou $f(z)=$ const sur chaque composant de la frontière $E$ d'un domaine $D_{\infty}$ contenant le point $z=\infty$.

Cas d'un domaine plan. Je vais démontrer le théorème suivant:

THÉoR̀̀m 1. Supposons $1^{\circ}$ que l'ensemble $E$ soit une courbe régulière de Jordan telle qu'on ait

$$
\left|\Theta\left(s^{\prime}\right)-\Theta\left(s^{\prime \prime}\right)\right| \leqslant A\left|s^{\prime}-s^{\prime \prime}\right|, \quad A=\text { const }
$$

(1) Il peut arriver qu'il y ait plusieurs systèmes de points extrémaux pour une valeur fixée de $n$ et $\lambda$, mais l'ensemble $E_{\lambda}$ est toujours le même. où $\Theta(s)$ désigne l'angle entre la droite tangente à $E$ et l'axe réel, $s-l a$ longueur de $E, 2^{\circ}$ que la fonction $f(z)$ remplisse sur $E$ la condition de Lipschitz

$$
\left|f\left(z^{\prime}\right)-f\left(z^{\prime \prime}\right)\right| \leqslant M\left|z^{\prime}-z^{\prime \prime}\right|
$$

Alors il existe un nombre $\lambda_{0}>0$ tel que la fonction $\frac{1}{\lambda} \log \Phi(z ; \lambda f)$ soit, pour chaque $\lambda, 0<\lambda \leqslant \lambda_{0}$, la solution du problème de Dirichlet pour le domaine $D$, où $D$ est l'intérieur de la courbe $E$.

Démonstration. Complétons la définition de la fonction $\frac{1}{\lambda} \log \Phi(z ; \lambda f)$ par ses valeurs limites $(*)$. La fonction $\frac{1}{\lambda} \log \Phi(z ; \lambda f)$ est sousharmonique dans tout le plan ouvert, car elle est égale à la différence d'une constante et d'un potentiel logarithmique (voir [5]). Désignons par $\varphi(z ; f)$ la fonction suivante:

$\varphi(z ; f)$ est égale à la solution du problème de Dirichlet pour la fonction $f(z)$ et le domaine $D$ lorsque $z \epsilon D$ et elle est égale à la même solution pour le domaine $D_{\infty}$ lorsque $z \epsilon D_{\infty}$

Soit $G(z ; \infty)$ la fonction de Green pour le domaine $D_{\infty}$ avec un pôle à l'infini. Posons $G(z ; \infty) \equiv 0$ pour $z \epsilon D$ et considérons la fonction

$$
\frac{1}{\lambda} G(z ; \infty)+\varphi(z ; f) \text {. }
$$

Pour que la fonction (7) soit sousharmonique dans tout le plan pour une valeur $\lambda_{0}>0$ il suffit qu'on ait

$$
\frac{1}{2 \pi r} \int_{K\left(z_{0} ; r\right)}\left[\frac{1}{\lambda_{0}} G(z ; \infty)+\varphi(z ; f)\right] d s \geqslant \varphi\left(z_{0} ; f\right),
$$

où $K\left(z_{0} ; r\right)$ est un cercle de centre au point quelconque $z_{0} \epsilon E$ et de rayon arbitraire $r$. L'inégalité (8) peut être représentée sous la forme suivante

$$
\frac{1}{\lambda_{0}} \geqslant \int_{\mathcal{K}\left(z_{0} ; r\right)}\left[\varphi\left(z_{0} ; f\right)-\varphi(z ; f)\right] d s / \int_{K\left(z_{0} ; r\right)} G(z ; \infty) d s .
$$

Selon les conditions (5), (6) et le théorème bien connu ( $[6]$, p. 456), la fonction $\varphi(z ; f)$ remplit dans tout le plan la condition de Lipschitz, donc on a

$$
\int_{K\left(z_{0} ; r\right)}\left|\varphi\left(z_{0} ; f\right)-\varphi(z ; f)\right| d s \leqslant B r^{2}, \quad B=\text { const. }
$$

Annales Polonici Mathernatici VII. 
D'autre part il résulte de l'hypothèse (5) qu'on a $\left(^{2}\right)$ (cf. [8], p. 664)

$$
\varliminf_{r \rightarrow 0} \frac{1}{r^{2}} \int_{\mathcal{K}\left(z_{0} ; r\right)} G(z ; \infty) d s \geqslant c>0, \quad C=\text { const. }
$$

Pour que l'inégalité (9) ait lieu il suffit qu'on ait

$$
\varlimsup_{r \rightarrow 0}\left\{\int_{K\left(z_{0} ; r\right)}\left[\varphi\left(z_{0} ; f\right)-\varphi(z ; f)\right] d s / \int_{\mathcal{K}\left(z_{0} ; r\right)} G(z ; \infty) d s\right\}<\infty .
$$

D'après (10), (11) et (12) il résulte qu'il existe un nombre $\lambda_{0}>0$ tel que pour chaque $\lambda, 0<\lambda \leqslant \lambda_{0}$ la fonction (7) est sousharmonique dans tout le plan ouvert.

Deux cas sont possibles: $E_{\lambda}=E$ ou $E_{\lambda} \neq E$. Lorsque $E_{\lambda}=E$ pour une valeur $\lambda>0$ la fonction $\frac{1}{\lambda} \log \Phi(z ; \lambda f)$ serait la solution cherchée. Soit $E_{\lambda} \neq E$ et $\lambda<\lambda_{0}$. Désignons par $D\left(E_{\lambda}\right)$ le domaine dont la frontière est l'ensemble $E_{\lambda}$ et formons la différence

$$
\frac{1}{\lambda} G(z ; \infty)+\varphi(z ; f)-\frac{1}{\lambda} \log \Phi(z ; \lambda f)
$$

pour $z \epsilon D\left(E_{\lambda}\right)$. Les fonctions $\frac{1}{\lambda} \log \Phi(z ; \lambda f)$ et $(7)$ sont égales sur $E_{\lambda}$, la fonction $\frac{1}{\lambda} \log \Phi(z ; \lambda f)$ est harmonique en dehors de $E_{\lambda}$ (le point $\infty$ exclu), la fonction (7) est sousharmonique en dehors de $\mathbb{E}_{\lambda}$. Le différence est donc une fonction sousharmonique en dehors de $E_{\lambda}$, égale à 0 sur $E_{\lambda}$ (et harmonique dans le voisinage du point $\infty$ ) alors

$$
\frac{1}{\lambda} G(z ; \infty)+\varphi(z ; f) \leqslant \frac{1}{\lambda} \log \Phi(z ; \lambda f), \quad z \in D\left(E_{\lambda}\right) .
$$

De cette inégalité et de (3) il résulte qu'on a $\frac{1}{\lambda} \log \Phi(z ; \lambda f)=f(z)$ pour chaque $z \in E$.

Remarque. $1^{\circ}$ Lorsque $0<\lambda \leqslant \lambda_{0}$ et le point $z \in D_{\infty}$ tend vers $z_{0} \epsilon E$, la fonction $\frac{1}{\lambda} \log \Phi(z ; \lambda f)$ tend vers $f\left(z_{0}\right) .2^{\circ}$ Le théorème 1 est vrai dans le cas où l'ensemble $E=\sum_{i=1}^{p} E_{i}, E_{j} E_{l}=0$ pour $j \neq k, j, k=$ $=1, \ldots, p$, est la frontière d'un domaine $D_{\infty}, E_{i}, i=1, \ldots, p$, étant la courbe de Jordan remplissant la condition (5).

(2) En chaque point $z_{6} E$ il existe une dérivée $d G / d n \geqslant o_{1}>0$, où $n$ désigne la
Cas d'un domaine dans l'espace. Soit $E$ un ensemble borné et fermé dans l'espace dont la capacité newtoniènne $d(E)$ est positive. Posons

$$
\omega_{\lambda}(P, Q)=\exp \left\{\lambda[f(P)+f(Q)]-\frac{1}{P Q}\right\}, \quad P, Q \in E,
$$

où $f(P)$ est la fonction continue sur $E$ et $P Q$ désigne la distance deș points $P$ et $Q$. Un système de $n$ points

$$
P_{\lambda}^{(n)}=\left\{P_{1}^{(n \lambda)}, P_{2}^{(n \lambda)}, \ldots, P_{n}^{(n \lambda)}\right\}
$$

de la frontière $E$ sera dit $n$-ième système de points extrémaux par rapport à la fonction (13) lorsqu'on a

$$
\prod_{1 \leqslant i<k \leqslant n} \omega_{\lambda}\left(P_{i}^{(n \lambda)}, P_{k}^{(n \lambda)}\right) \geqslant \prod_{1 \leqslant i<k \leqslant n} \omega_{\lambda}\left(Q_{i}, Q_{k}\right)
$$

pour chaque système de $n$ points $Q^{\left(n_{j}\right.} \in E$.

Dans le travail [3] j'ai prouvé: $1^{\circ}$ Pour chaque point $P \notin E$ il existe une limite

$$
\lim _{n \rightarrow \infty} \frac{1}{n} \sum_{k=1}^{n} \frac{1}{P P_{k}^{(n \lambda)}}=v_{\lambda}(P) .
$$

On peut représenter la fonction $v_{\lambda}(P)$ par l'intégrale

$$
v_{\lambda}(P)=\int_{E} \frac{d \mu_{\lambda}(Q)}{P Q},
$$

où $\mu_{\lambda}$ désigne la répartition limite de la masse unité sur $E$. Cette répartition est définie par les points extrémaux $P_{\lambda}^{(n)} .2^{\text {o }}$ Pour chaque point $P \epsilon E$, à l'exception d'un ensemble de capacité 0 , on a

$$
\begin{gathered}
\int_{L} \frac{d \mu_{\lambda}(Q)}{P Q} \geqslant \lambda f(P)+\gamma_{\lambda}, \\
\gamma_{\lambda}=\frac{1}{d\left(E_{\lambda}\right)}-\lambda \int_{E_{\lambda}} f(Q) d \mu_{\lambda}(Q) .
\end{gathered}
$$

$E_{\lambda}$ est le noyau de la masse relatif à la distribution $\mu_{\lambda}, d\left(E_{\lambda}\right)$ est la capacité de l'ensemble $E_{\lambda}$ et $\mu_{\lambda}$ la distribution d'équilibre de la masse unité sur $E_{\lambda} .3^{\circ}$ La fonction $\frac{1}{\lambda} v_{\lambda}(P)$ est la solution du problème de Dirichlet pour la fonction $f(P)+\gamma_{\lambda} / \lambda$ et le domaine $D_{\lambda}$ dont la frontière est l'ensemble $E_{\lambda}$. A l'intini on a $\frac{1}{\lambda} v_{\lambda}(\infty)=0$. 
La fonction (14) est surharmonique dans tout l'espace. Désignons par $\psi(P ; f)$ la fonction suivante:

$\psi(P ; f)$ est la solution du problème de Dirichlet pour la domaine borné $D$ (dont la frontière est l'ensemble $E$ ) avec la valeur $f(P)$ sur $E$ lorsque $P \in D$ et

$\psi(P ; f)$ est égale à la même solution avec la valeur 0 à l'infini lorsque $P \in D_{\infty}$, où $D_{\infty}$ est le domaine non borné dont la frontière est l'ensemble $E$.

Soit de même $\varphi_{\lambda}(P)$ la solution du problème de Dirichlet pour le

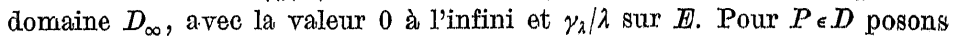
$\varphi_{\lambda}(P) \equiv \gamma_{\lambda} / \lambda$.

Pour que la fonction $\psi(P ; f)+\varphi_{\lambda}(P)$ soit surharmonique dans tout l'espace, il suffit qu'on ait

$$
\frac{1}{4 \pi r^{2}} \int_{\Sigma(Q ; r)}\left[\psi(P ; f)+\varphi_{\lambda}(P)\right] d \sigma_{p} \leqslant f(Q)+\frac{\gamma_{\lambda}}{\lambda}
$$

pour chaque point $Q \in E$ et chaque sphère $K(Q ; r)$ de centre $Q$ et de rayon $r$.

Lorsqu'il existe un nombre $\lambda_{0}>0$ tel que l'inégalité (16) soit satisfaite, on a $E_{\lambda}=E$ pour $0<\lambda \leqslant \lambda_{0}$, car dans lo cas contraire la fonction $\frac{1}{\lambda} v_{\lambda}(P)$ serait harmonique en dehors de l'ensemble $E_{\lambda}$, done (cf. la démonstration $\mathrm{du}$ théorème 1.)

$$
\frac{1}{\lambda} v_{\lambda}\left(P_{0}\right) \leqslant \psi\left(P_{0} ; f\right)+\varphi_{\lambda}\left(P_{0}\right)=f\left(P_{0}\right)+\frac{\gamma_{\lambda}}{\lambda}, \quad P_{0} \in E-E_{\lambda} .
$$

De cette inégalité et de (15) on tire $\frac{1}{\lambda} v_{\lambda}(P)=f(P)+\frac{\gamma_{\lambda}}{\lambda}$ pour $P \epsilon E$. Alors la fonction $\frac{1}{\lambda} v_{\lambda}(P)$ est la solution du problème de Dirichlet pour le domaine $D$ (et $D_{\infty}$ ) arec la valeur $f(P)+\gamma_{\lambda} / \lambda$ sur $E$ (et 0 à l'infini).

THÉoREme 2. Soit $\boldsymbol{E}$ la frontière commune de deus domaines $D$ et $\mathrm{D}_{\infty}$. Supposons $1^{\circ}$ que $E$ soit la surface de Liapounoff, $2^{\circ}$ que la fonotion $f(P)$ remplisse la condition de Lipschitz. Alors il existe un nombre $\lambda_{0}>0$ tel que la condition (16) soit satisfaite.

Démonstration. D'après $1^{\circ}, 2^{\circ}$ et un théorème connu (voir [7], p. 21,3$)$ on a

$$
|\psi(P ; f)-f(Q)| \leqslant B|P Q|
$$

dans tout l'espace. D'autre part pour $\lambda \rightarrow 0$ on a $\gamma_{\lambda} / \lambda \rightarrow \infty$ car $d\left(\mathbb{B}_{\lambda}(\rightarrow\right.$ $\rightarrow d(E)>0$ (voir [3]). II suffit de prouver l'inégalité (16) pour de petites valeurs $d u$ rayon $r$. Mais, pour $r$ suffisamment petit, on $a\left(^{3}\right)$

$$
\frac{1}{4 \pi r^{2}} \int_{K(Q ; r)} \int_{\lambda} \varphi_{\lambda}(P) d \sigma_{p}<\frac{\gamma_{\lambda}}{\lambda}-A_{\lambda} \cdot r,
$$

où la constante $A_{\lambda}$ depéndant de $\lambda$ est $>B$ pour $\lambda \leqslant \lambda_{0}$; done (17) et (18) entraînent l'inégalité (16).

\section{Travaux cités}

[1] F. Leja, Une méthode élémentaire de résolution du problème de Dirichlet dans le plan, Ann. de la Soc. Polon. de Math. 23 (1950), p. 230-245.

[2] M. Inoue, Sur un procédé pour construire la solution du problème de Dirichlet, Proc. Imp. Acad. Tokyo 14 (1936), p. 368-372.

3] J. Górski, Méthode des points extrémaux de résolution du problème de Diriohlet dans l'espace, Ann. Pol. Math. 1 (1955), p. 218-229.

[4] - Sur certaines fonotions harmoniques jouissant des propriétés extrémales par rapport à un ensemble, Ann. de la Soc. Polon. de Math. 23 (1950), p. 259-271.

[5] - Sur certaines propriétés des points extrémaux liés à un domaine plan, Ann. Pol. Math. 3 (1956), p. $32-36$.

[6] Г. М. Г о л у в и н, Геометрическал теория функиий комплексіного переменного, Москва 1952 .

[7] N. M. Giunter, Teoria potencjału, Warszawa 1957, str. 328.

[8] В. И. Смирн ов, Курс высшей математики IV, Москва 1949.

\section{Regu par la Rédaction le 11.4.1958} $A_{\lambda}^{*}>0$.

$\left(^{3}\right)$ En chaque point $Q_{6} E$ il existe une dérivée normale $d \varphi_{\lambda}(P) / d n \leqslant-A_{\lambda}^{*}$ 